\title{
LATTICE EMBEDDINGS OF ABELIAN PRIME POWER GROUPS
}

\author{
ROLAND SCHMIDT
}

(Received 10 January 1996; revised 29 July 1996)

Communicated by R. Howlett

\begin{abstract}
We solve the following problem which was posed by Barnes in 1962. For which abelian groups $G$ and $H$ of the same prime power order is it possible to embed the subgroup lattice of $G$ in that of $H$ ? It follows from Barnes' results and a theorem of Herrmann and Huhn that if there exists such an embedding and $G$ contains three independent elements of order $p^{2}$, then $G$ and $H$ are isomorphic. This reduces the problem to the case that $G$ is the direct product of cyclic $p$-groups only two of which have order larger than $p$. We determine all groups $H$ for which the desired embedding exists.
\end{abstract}

1991 Mathematics subject classification (Amer. Math. Soc.): primary 20D30; secondary $20 \mathrm{~K} 01$.

\section{Introduction}

We want to solve the following problem. Given two abelian groups $G$ and $H$ of the same prime power order, when does there exist an embedding (that is, a monomorphism) of the subgroup lattice $L(G)$ of $G$ in that of $H$ ?

This problem was studied and partly solved by Barnes [1] already in 1962 . He showed that if $H$ is elementary abelian, then $L(G)$ can be embedded in $L(H)$ if and only if $G$ does not contain a subgroup of type $(3,3,2)$. Recall that a finite abelian $p$-group $G$ is a direct product of cyclic groups of order $p^{\lambda_{1}}, \ldots, p^{\lambda_{r}}$ where $\lambda_{1} \geq \cdots \geq \lambda_{r} \geq 1$, and then the $r$-tuple $\left(\lambda_{1}, \ldots, \lambda_{r}\right)$ is called the type of $G$. In the general case, Barnes showed further that if $G$ has a subgroup of this type and $L(G)$ is embedded in $L(H)$, then $G$ and $H$ are isomorphic; but he left the problem open for groups having no such subgroups.

Unfortunately, these results are not quite correct. They are inconsistent with a theorem of Herrmann and Huhn saying that a certain lattice law holds in the subgroup lattice of an elementary abelian $p$-group but not in that of an abelian $p$-group of type

(C) 1997 Australian Mathematical Society $0263-6115 / 97 \$ A 2.00+0.00$ 
$(2,2,2)$ (see [2, Theorem 5]). As Barnes told me, this was realized in 1980 by Sheila Oates-Williams who also located and corrected his error: in the proof of Lemma 6.2 of his paper he uses an argument that a certain set of $n$ equations modulo $p^{2}$ implies two sets of $n$ equations modulo $p$ which, however, is not the case.

It is not difficult to see that the theorem of Herrmann and Huhn has just the effect of replacing the group of type $(3,3,2)$ in Barnes' results by the group of type $(2,2,2)$. So we obtain the following theorems in which we call an embedding of $L(G)$ in $L(H)$ an $L$-embedding of $G$ in $H$, for short.

THEOREM A. There exists an L-embedding of the abelian p-group $G$ in the elementary abelian group if and only if $G$ has no subgroup of type $(2,2,2)$.

An easy consequence of this is

THEOREM B. Let $G$ and $H$ be abelian groups of the same prime power order. Suppose $G$ has a subgroup of type $(2,2,2)$ and that $\varphi$ is an L-embedding of $G$ in $H$. Then $X^{\varphi} \simeq X$ for every subgroup $X$ of $G$; in particular, $H \simeq G$.

This theorem reduces the general problem to groups of type $\left(\lambda_{1}, \ldots, \lambda_{r}\right)$ where $r \leq 2$ or $\lambda_{3}=1$. The subgroup lattice of a cyclic group of order $p^{n}$ is a chain of length $n$ and therefore can be embedded in $L(H)$ for any group $H$ of order $p^{n}$. So we may assume that $r \geq 2$ and the following result completes the solution of our problem.

THEOREM C. Let $n \geq k \geq 1$ and $r \geq 2$. Suppose that $G=A \times B \times C_{3} \times \cdots \times C_{r}$ where $A$ and $B$ are cyclic of order $p^{n}$ and $p^{k}$, respectively, and $\left|C_{i}\right|=p$ for $i=$ $3, \ldots, r$; it is understood that $G=A \times B$ if $r=2$. Then $G$ has an $L$-embedding in an abelian group $H$ of the same order if and only if there exist subgroups $U, V, W_{i}$ of $H$ such that $H=U \times V \times W_{3} \times \cdots \times W_{r},|U|=p^{n},|V|=p^{k},\left|W_{i}\right|=p$ for $i=3, \ldots, r$, and one of the following holds.

(i) $k=1$.

(ii) $k \geq 2, n=\lambda k+t$ where $\lambda \in \mathbb{N}$ and $0 \leq t<k, V$ is elementary abelian and $U$ has type $\left(\alpha_{1}, \ldots, \alpha_{s}\right)$ where $\alpha_{1} \leq \lambda+1$ and $\alpha_{t+1} \leq \lambda$.

(iii) $U \simeq G_{n, s}$ and $V \simeq G_{k, s}$ for some integer s such that $1 \leq s<k$.

Here, if $1 \leq s \leq m$, the group $G_{m, s}$ is defined as follows: write $m=\mu s+t$ with $\mu \in \mathbb{N}$ and $0 \leq t<s$; then $\mu, t$ are uniquely determined by $m, s$ and we let $G_{m, s}$ be the abelian group of type $\left(\mu_{1}, \ldots, \mu_{s}\right)$ where $\mu_{i}=\mu$ for $i>t$ and $\mu_{i}=\mu+1$ for $i \leq t$. Then $G_{m, s}$ is a group of order $p^{m}$ with $s$ generators; in particular, $G_{m, 1}$ is the cyclic and $G_{m, m}$ the elementary abelian group of order $p^{m}$.

Since neither Barnes nor Oates-Williams published a corrected version of Barnes' results, we shall give short proofs of Theorems A and B in Section 1. The proof of 
Theorem C will occupy Sections 2 and 3; crucial for this is the concept of a smooth group introduced in 2.1 .

All groups considered are finite, the notation is standard (see $[1,4]$ ), except that we write $A \cup B$ for the group generated by the subgroups $A$ and $B$ of the group $X$.

\section{Barnes' results}

In the whole paper, $p$ is a prime and $G$ and $H$ are abelian $p$-groups of the same order. A lattice embedding (which we abbreviate to $L$-embedding) of $G$ in $H$ is an embedding of $L(G)$ in $L(H)$, and this is defined to be an injective mapping $\varphi$ of $L(G)$ into $L(H)$ such that $X^{\varphi}$ covers $Y^{\varphi}$ for all $X, Y \in L(G)$ where $X$ covers $Y$. Barnes shows [1, Corollary 1.3] that in our case (of abelian groups) this is equivalent to $\varphi$ being a lattice monomorphism, that is, an injective map satisfying

(1) $(X \cup Y)^{\varphi}=X^{\varphi} \cup Y^{\varphi} \quad$ and $\quad(X \cap Y)^{\varphi}=X^{\varphi} \cap Y^{\varphi} \quad$ for all $X, Y \in L(G)$.

We need the following lattice polynomials introduced by Herrmann and Huhn. For elements $A, B, C, D$ of a lattice $L$, we define inductively

$$
\begin{aligned}
f_{1}(A, B, C, D)= & (A \cup B) \cap(C \cup D) \\
f_{n+1}(A, B, C, D)= & \left.\left(\left(f_{n}(A, B, C, D) \cup f_{1}(A, C, B, D)\right) \cap(B \cup C)\right) \cup D\right) \\
& \cap(A \cup B) .
\end{aligned}
$$

LEMMA 1.1 (Herrmann and Huhn [2]). Let $X=A \times B \times C$ with isomorphic abelian groups $A, B, C$. Suppose that $\mu: A \rightarrow B$ and $v: A \rightarrow C$ are isomorphisms and let $D=\left\{a a^{\mu} a^{\nu} \mid a \in A\right\}$. Then for all $n \in \mathbb{N}$,

$$
f_{n}(A, B, C, D)=\left\{a\left(a^{n}\right)^{\mu} \mid a \in A\right\} .
$$

ProOF. We use induction on $n$. Since $A \cup B=A B=\{a b \mid a \in A, b \in B\}$ and $C \cup D=\left\{a a^{\mu} c \mid a \in A, c \in C\right\}$, we have $f_{1}(A, B, C, D)=\left\{a a^{\mu} \mid a \in A\right\}$ and the assertion holds for $n=1$. If we write $F=f_{n}(A, B, C, D) \cup f_{1}(A, C, B, D)$, the induction assumption yields that

$$
F=\left\{a\left(a^{n}\right)^{\mu} \mid a \in A\right\} \cdot\left\{b b^{\nu} \mid b \in A\right\}=\left\{a b\left(a^{n}\right)^{\mu} b^{\nu} \mid a, b \in A\right\} .
$$

An element $a b\left(a^{n}\right)^{\mu} b^{v}$ of $F$ lies in $B \cup C$ if and only if $b=a^{-1}$. Therefore

$$
\begin{aligned}
(F \cap(B \cup C)) \cup D & =\left\{\left(a^{n}\right)^{\mu}\left(a^{-1}\right)^{v} \mid a \in A\right\} \cdot\left\{c c^{\mu} c^{\nu} \mid c \in A\right\} \\
& =\left\{c\left(a^{n} c\right)^{\mu}\left(a^{-1} c\right)^{\nu} \mid a, c \in A\right\}
\end{aligned}
$$


and such an element lies in $A \cup B$ if and only if $a=c$. Thus

$$
f_{n+1}(A, B, C, D)=\left\{a\left(a^{n+1}\right)^{\mu} \mid a \in A\right\},
$$

as desired.

We can now prove Theorems A and B stated in the introduction.

PROOF OF THEOREM A. If $G$ has no subgroup of type $(2,2,2)$, then its type is of the form $\left(\lambda_{1}, \ldots, \lambda_{r}\right)$ with $r \leq 2$ or $\lambda_{3}=1$. By [1, Lemma 6.1], $G$ has an $L$-embedding in the elementary abelian group.

Since an $L$-embedding of a group induces an $L$-embedding of every subgroup, to prove the converse, we only have to show that there is no $L$-embedding of a group $G$ of type $(2,2,2)$ in an elementary abelian group $H$ of order $p^{6}$. So suppose, for a contradiction, that $\varphi: L(G) \rightarrow L(H)$ is such an embedding, let $G=A \times B \times C$ where $A, B, C$ are cyclic of order $p^{2}$ and let $\mu, v$, and $D$ be as in Lemma 1.1. Then $D$ is a complement to $A \cup B, A \cup C$, and to $B \cup C$ in $G$.

By (1), $H=A^{\varphi} \times B^{\varphi} \times C^{\varphi}$ and $D^{\varphi}$ is a complement to $A^{\varphi} \cup B^{\varphi}, A^{\varphi} \cup C^{\varphi}$, and to $B^{\varphi} \cup C^{\varphi}$ in $H$. It follows that the projection of $D^{\varphi}$ into $A^{\varphi}$ is surjective, that the maps $\sigma: A^{\varphi} \rightarrow B^{\varphi}$ and $\tau: A^{\varphi} \rightarrow C^{\varphi}$ mapping the first component of an element of $D^{\varphi}$ to its second and third component, respectively, are isomorphisms and that $D^{\varphi}=\left\{a a^{\sigma} a^{\tau} \mid a \in A^{\varphi}\right\}$.

By 1.1, $f_{p}(A, B, C, D)=\left\{a\left(a^{p}\right)^{\mu} \mid a \in A\right\} \neq A$ and by (1) and 1.1,

$$
f_{p}(A, B, C, D)^{\varphi}=f_{p}\left(A^{\varphi}, B^{\varphi}, C^{\varphi}, D^{\varphi}\right)=\left\{a\left(a^{p}\right)^{\sigma} \mid a \in A^{\varphi}\right\}=A^{\varphi}
$$

since $H$ has exponent $p$; but $\varphi$ is injective. This contradiction shows that there is no $L$-embedding of $G$ in $H$.

Proof OF THEOREM B. We use induction on $|G|$. Let $X \leq G$. Then we have $X=X_{1} \times \cdots \times X_{r}$ with cyclic subgroups $X_{i}$ and by (1), $X^{\varphi}=X_{1}^{\varphi} \times \cdots \times X_{r}^{\varphi}$. So if $X_{i}^{\varphi} \simeq X_{i}$ for all $i$, then $X^{\varphi} \simeq X$. Thus we may assume that $X$ is cyclic and that $|X|=p^{k}$ where $k \geq 2$. Since $G$ contains a subgroup of type $(2,2,2)$, there exist cyclic subgroups $Y$ and $Z$ of $G$ such that $G_{0}=X \times Y \times Z$ is a subgroup of type $(k, 2,2)$ of $G$. If $k=2$ and $X^{\varphi}$ were not cyclic, then $G_{0}^{\varphi}=X^{\varphi} \times Y^{\varphi} \times Z^{\varphi}$ would not be of type $(2,2,2)$ and hence, by Theorem $A$, would have an $L$-embedding in an elementary abelian group. But then also $G_{0}$ would have such an $L$-embedding, contradicting Theorem A. Thus $X^{\varphi} \simeq X$ in this case.

Finally, assume that $k \geq 3$ and let $A$ be the maximal subgroup of $X$. Then $A \times Y \times Z$ and $G / \Omega(X)$ both contain subgroups of type $(2,2,2)$ and hence by induction, $A^{\varphi}$ and $X^{\varphi} / \Omega(X)^{\varphi}$ are cyclic. Since $k \geq 3, \Omega(X)<A$ and hence $\Omega(X)^{\varphi} \leq \Phi\left(A^{\varphi}\right)$; furthermore $\Phi\left(A^{\varphi}\right) \leq \Phi\left(X^{\varphi}\right)$ since $X^{\varphi}$ is a $p$-group (see [3, p. 273]). Thus $X^{\varphi} / \Phi\left(X^{\varphi}\right)$ is cyclic; hence $X^{\varphi}$ is cyclic and $X^{\varphi} \simeq X$. 


\section{Smooth groups}

In this section we shall prove that if $G$ is of type $\left(\lambda_{1}, \ldots, \lambda_{r}\right)$ with $r \leq 2$ or $\lambda_{3}=1$ and $L(G)$ can be embedded in $L(H)$, then $H$ has the structure given in Theorem C. Basic for this is the following concept.

DEFINITION 2.1. Let $p$ be a prime, $n, k \in \mathbb{N}$, let $G$ be an abelian group of order $p^{n}$ and suppose that $X_{i} \leq G$ are such that

$$
1=X_{0}<X_{1}<\cdots<X_{n}=G
$$

thus $\left|X_{i}\right|=p^{i}$ for $i=0, \ldots, n$.

(a) The chain (2) is called $k$-smooth if for every $j \in\{1, \ldots, k\}$, we have $X_{j} \simeq$ $X_{i+j} / X_{i}$ for all $i=1, \ldots, n-j$.

(b) The group $G$ is called $k$-smooth if there exists a $k$-smooth chain (2) in $G$; in this case, the isomorphism type of the group $X_{k}$ is called the $k$-type of $G$.

(c) The chain (2) or the group $G$ is called smooth if it is $n$-smooth; that is, if it is $k$-smooth for every $k \in \mathbb{N}$.

Certainly, cyclic and elementary abelian $p$-groups are smooth and it is also clear that all the subgroups and factor groups appearing in a smooth chain are smooth. More precisely, we have the following inheritance property.

LEMMA 2.2. If (2) is a $k$-smooth chain, $0 \leq s<t \leq n$ and $1 \leq j \leq k$, then the chain

$$
1=X_{s} / X_{s}<X_{s+1} / X_{s}<\cdots<X_{t} / X_{s}
$$

is $j$-smooth. In particular, if (2) is smooth, then so is (3).

The significance of the concept of smooth groups for our problem may be seen from the following result.

LEMMA 2.3. Let $n \geq k \geq 1$ and $r \geq 2$. Let $G=A \times B \times C_{3} \times \cdots \times C_{r}$, where $A$ and $B$ are cyclic of order $p^{n}$ and $p^{k}$, respectively, and $\left|C_{i}\right|=p$ for $i=3, \ldots, r$. If $\varphi$ is an L-embedding of $G$ in the abelian group $H$, then $H=A^{\varphi} \times B^{\varphi} \times C_{3}^{\varphi} \times \cdots \times C_{r}^{\varphi}$ where $A^{\varphi}$ and $B^{\varphi}$ are $k$-smooth of $k$-type $B^{\varphi}$. More precisely, if $A_{i}$ and $B_{i}$ are the subgroups of order $p^{i}$ of $A$ and $B$, respectively, then $1=A_{0}^{\varphi}<\cdots<A_{n}^{\varphi}=A^{\varphi}$ and $1=B_{0}^{\varphi}<\cdots<B_{k}^{\varphi}=B^{\varphi}$ are $k$-smooth chains and $B^{\varphi} \simeq A_{k}^{\varphi}$.

PROOF. By (1), $H=A^{\varphi} \times B^{\varphi} \times C_{3}^{\varphi} \times \cdots \times C_{r}^{\varphi}$ since every subgroup of $H$ is normal. We show that the chain $1=A_{0}^{\varphi}<\cdots<A_{n}^{\varphi}=A^{\varphi}$ is $k$-smooth. For this let $1 \leq j \leq k$ 
and $0 \leq i \leq n-j$ and put $R=A_{i+j} \times B_{j}$. Then $R / A_{i}=A_{i+j} / A_{i} \times B_{j} A_{i} / A_{i}$ is a direct product of two cyclic groups of order $p^{j}$. By [4, Theorem 1.6.2] there exists a diagonal $S / A_{i}$ in this group; that is, $S \cap A_{i+j}=A_{i}=S \cap B_{j} A_{i}$ and $S \cup A_{i+j}=R=S \cup B_{j} A_{i}$. By (1), $S^{\varphi} / A_{i}^{\varphi}$ is a diagonal in $R^{\varphi} / A_{i}^{\varphi}=A_{i+j}^{\varphi} / A_{i}^{\varphi} \times B_{j}^{\varphi} A_{i}^{\varphi} / A_{i}^{\varphi}$ and [4, Theorem 1.6.2] now implies that $A_{i+j}^{\varphi} / A_{i}^{\varphi} \simeq B_{j}^{\varphi} A_{i}^{\varphi} / A_{i}^{\varphi} \simeq B_{j}^{\varphi}$. For $i=0$ we obtain $A_{j}^{\varphi} \simeq B_{j}^{\varphi}$ and hence $A_{i+j}^{\varphi} / A_{i}^{\varphi} \simeq A_{j}^{\varphi}$ for arbitrary $i$. Thus our chain is $k$-smooth and $A_{k}^{\varphi} \simeq B_{k}^{\varphi}=B^{\varphi}$. In the same way we show that the chain $1=B_{0}^{\varphi}<\cdots<B_{k}^{\varphi}=B^{\varphi}$ is $k$-smooth.

The above lemma in particular says that $B^{\varphi}$ is smooth. Therefore our next aim is to determine all smooth groups. For this we need that every smooth chain of $G$ contains all the groups

$$
\Omega_{m}(G):=\left\{x \in G \mid x^{p^{m}}=1\right\} \text { and } G^{p^{m}}:=\left\{x^{p^{m}} \mid x \in G\right\} .
$$

LEMMA 2.4. Let $1=X_{0}<\cdots<X_{n}=G$ be a smooth chain in an abelian $p$ group $G$. For every $m \in \mathbb{N}$ there exist integers $i$ and $j$ such that $\Omega_{m}(G)=X_{i}$ and $G^{p^{m}}=X_{j}$.

PROOF. Since $\Omega_{m}(G) / \Omega_{1}(G)=\Omega_{m-1}\left(G / \Omega_{1}(G)\right)$ and $G^{p^{m}}=\left(G^{p}\right)^{p^{m-1}}$, Lemma 2.2 and an obvious induction yield that we only have to show the assertion for $m=1$. To do this we use induction on $|G|$. By 2.2 and the induction assumption there exist integers $s$ and $t$ such that $\Omega\left(G / X_{1}\right)=X_{s} / X_{1}$ and $X_{n-1}^{p}=X_{t}$.

If $X_{s}<G$, then again by induction, $\Omega(G)=\Omega\left(X_{s}\right)=X_{i}$ for some $i \in \mathbb{N}$. And if $X_{s}=G$, then $X_{n-1} \simeq G / X_{1}$ is elementary abelian and hence $\Omega(G)=X_{n-1}$ or $\Omega(G)=G=X_{n}$.

Similarly, if $X_{t} \neq 1$, the induction assumption implies that $G^{p} / X_{t}=\left(G / X_{t}\right)^{p}=$ $X_{j} / X_{t}$ for some integer $j$ and hence $G^{p}=X_{j}$. And if $X_{t}=1$, then $G / X_{1} \simeq X_{n-1}$ is elementary abelian and hence $G^{p}=X_{1}$ or $G^{p}=1=X_{0}$, as desired.

THEOREM 2.5. The abelian p-group of type $\left(\lambda_{1}, \ldots, \lambda_{r}\right)$ is smooth if and only if $\lambda_{1}-\lambda_{r} \leq 1$.

ProOF. Write $G=A_{1} \times \cdots \times A_{r}$ with cyclic groups $A_{i}$ of order $p^{\lambda_{i}}$ where $\lambda_{1} \geq \cdots \geq \lambda_{r} \geq 1$ and put $\lambda=\lambda_{1}$.

First assume that $G$ is smooth and let $1=X_{0}<\cdots<X_{n}=G$ be a smooth chain. If $\lambda \leq 2$, then clearly $\lambda_{1}-\lambda_{r} \leq 1$, so assume further that $\lambda \geq 3$. For every $\mu \in \mathbb{N}$,

$$
\Omega_{\mu}(G)=\Omega_{\mu}\left(A_{1}\right) \times \cdots \times \Omega_{\mu}\left(A_{r}\right)
$$

and hence $p^{j}:=\left|\Omega_{\lambda-1}(G) / \Omega_{\lambda-2}(G)\right| \leq p^{r}$ with equality if and only if $\lambda_{r} \geq \lambda-1$. By Lemma 2.4, $\Omega(G)=X_{r}$ and there exists $i \in \mathbb{N}$ such that $\Omega_{\lambda-2}(G)=X_{i}$ and 
$\Omega_{\lambda-1}(G)=X_{i+j}$. Then $\Omega\left(G / X_{i}\right)=X_{i+j} / X_{i}$ and hence $X_{i+j+1} / X_{i}$ is not elementary abelian. Since the chain is smooth, $X_{i+j+1} / X_{i} \simeq X_{j+1}$ and therefore $X_{j+1} \not X_{r}$. It follows that $j=r$ and so $\lambda_{r} \geq \lambda-1$, as desired.

Now suppose that, conversely, $\lambda_{r} \geq \lambda-1$. Then there exists $s \leq r$ such that $\lambda_{i}=\lambda$ for $1 \leq i \leq s$ and $\lambda_{i}=\lambda-1$ for $s+1 \leq i \leq r$; thus $n=r(\lambda-1)+s$. Since cyclic groups are smooth, we may assume that $r \geq 2$.

For $1 \leq i \leq n$, write $i=r j+k$ where $0 \leq j \leq \lambda-1$, and where $0<k \leq r$ if $j<\lambda-1,0<k \leq s$ if $j=\lambda-1$, and define

$$
X_{i}=\Omega_{j+1}\left(A_{1}\right) \times \cdots \times \Omega_{j+1}\left(A_{k}\right) \times \Omega_{j}\left(A_{k+1}\right) \times \cdots \times \Omega_{j}\left(A_{r}\right) .
$$

We prove by induction on $|G|$ that $1=X_{0}<\cdots<X_{n}=G$ is a smooth chain. First of all, clearly, $X_{i}<X_{i+1}$ for all $i$ and $\left|X_{i}\right|=p^{i}$. For $U \leq G$ we write $\bar{U}=U X_{1} / X_{1}$, let $\bar{A}_{r+1}:=\bar{A}_{1}$ and define $Y_{i}$ with respect to the decomposition $\bar{G}=\bar{A}_{2} \times \cdots \times \bar{A}_{r} \times \bar{A}_{r+1}$ as we defined $X_{i}$ in (4). Then for $i=r j+k \leq n-1$ as above, we obtain that

$$
Y_{i}=\Omega_{j+1}\left(\bar{A}_{2}\right) \times \cdots \times \Omega_{j+1}\left(\bar{A}_{k+1}\right) \times \Omega_{j}\left(\bar{A}_{k+2}\right) \times \cdots \times \Omega_{j}\left(\bar{A}_{r+1}\right) .
$$

Since $\Omega_{j}\left(\bar{A}_{r+1}\right)=\Omega_{j+1}\left(A_{1}\right) / X_{1}$, we have $Y_{i}=X_{i+1} / X_{1}$ for all $i$; this is clear if $k<r$, and for $k=r$ it follows since then $i+1=r(j+1)+1$. By the induction assumption, $1=Y_{0}<\cdots<Y_{n-1}=G / X_{1}$ is a smooth chain. Furthermore $Y_{i}$ and $X_{i}$ both are the direct product of $k$ cyclic groups of order $p^{j+1}$ and $r-k$ cyclic groups of order $p^{j}$. Therefore $Y_{i} \simeq X_{i}$ and hence for $i=1, \ldots, n-1$ and $t=1, \ldots, n-i$, we obtain that $X_{t+i} / X_{t} \simeq Y_{t+i-1} / Y_{t-1} \simeq Y_{i} \simeq X_{i}$. Thus the chain $1=X_{0}<\cdots<X_{n}=G$ is smooth.

COROLLARY 2.6. If $1 \leq r \leq n$, then the group $G_{n, r}$ defined in the Introduction is the unique smooth abelian group of order $p^{n}$ with $r$ generators.

PROOF. Let $G$ be an abelian group of order $p^{n}$ with $r$ generators, that is, of type $\left(\lambda_{1}, \ldots, \lambda_{r}\right)$. By Theorem $2.5, G$ is smooth if and only if there exist integers $s, \lambda$ with $0 \leq s<r$ such that $\lambda_{i}=\lambda$ for $i>s$ and $\lambda_{i}=\lambda+1$ for $i \leq s$. Since $n=\sum_{i=1}^{r} \lambda_{i}$, this implies that $n=r \lambda+s$. Thus $G$ is smooth if and only if $G \simeq G_{n, r}$.

In view of Lemma 2.3 we still have to determine all $k$-smooth groups of order $p^{n}$ for $k<n$. If $k=1$, every group of order $p^{n}$ is $k$-smooth. So we may assume that $k \geq 2$ and show first that we don't get any new groups if the $k$-type is not elementary abelian. Note that every $k$-type is the isomorphism type of a smooth group of order $p^{k}$ and therefore of one of the groups $G_{k, r}(r \leq k)$ appearing in Corollary 2.6.

THEOREM 2.7. Let $1 \leq r<k \leq n$. The abelian group $G$ of order $p^{n}$ is $k$-smooth of $k$-type $G_{k, r}$ if and only if $G$ is the smooth group $G_{n, r}$ of order $p^{n}$ with $r$ generators. 
PROOF. If $G=G_{n, r}$ and $1=X_{0}<\cdots<X_{n}=G$ is a smooth chain, then by 2.2, $G$ is $k$-smooth and $X_{k}$ is smooth. By $2.4, \Omega(G)=X_{r} \leq X_{k}$ and hence $\left|\Omega\left(X_{k}\right)\right|=p^{r}$. By Corollary 2.6, $X_{k} \simeq G_{k, r}$.

Conversely, assume that $1=X_{0}<\cdots<X_{n}=G$ is a $k$-smooth chain and $X_{k} \simeq G_{k, r}$. By 2.2 and 2.4, $\Omega\left(X_{k}\right)=X_{r}$ and since $r<k$, it follows from 2.6 that $X_{r+1} \simeq G_{r+1, r}$. Thus $G$ is $(r+1)$-smooth of $(r+1)$-type $G_{r+1, r}$ and it suffices to prove the following special case of our assertion.

(5) If $1 \leq r<n,|G|=p^{n}$ and $G$ is $(r+1)$-smooth of $(r+1)$-type $G_{r+1, r}$, then $G \simeq G_{n, r}$.

To prove this, we use induction on $n$. The assertion is clearly true for $n=r+1$. So assume it holds for $r+1 \leq m<n$ and let $1=Y_{0}<\cdots<Y_{n}=G$ be an $(r+1)$ smooth chain with $Y_{r+1} \simeq G_{r+1, r}$. Then $Y_{n-1} \simeq G_{n-1, r}$ and by $2.4, \Omega\left(Y_{n-1}\right)=Y_{r}$. If $\Omega(G)>Y_{r}$ and $x \in \Omega(G) \backslash Y_{r}$, then $x \notin Y_{n-1}$ and hence $G=Y_{n-1} \times\langle x\rangle$. Since $Y_{n-1} / Y_{n-1-r} \simeq Y_{r}$, it would follow that $G / Y_{n-1-r}$ is elementary abelian of order $p^{r+1}$; but $G / Y_{n-1-r} \simeq Y_{r+1}$ is not elementary abelian. This contradiction shows that $\Omega(G)=Y_{r} ;$ let $\left(\lambda_{1}, \ldots, \lambda_{r}\right)$ be the type of $G$.

Now $|G / \Omega(G)|=p^{n-r}$. If $n \leq 2 r$, then $G / \Omega(G) \simeq Y_{n-r} \leq Y_{r}$ is elementary abelian and hence $\operatorname{Exp} G \leq p^{2}$; by $2.5, G$ is smooth. So assume that $n>2 r$. Then the induction assumption implies that $G / \Omega(G) \simeq G_{n-r, r}$. It follows that $\lambda_{r} \geq 2$ and $G / \Omega(G)$ is smooth of type $\left(\lambda_{1}-1, \ldots, \lambda_{r}-1\right)$. By $2.5,1 \geq\left(\lambda_{1}-1\right)-\left(\lambda_{r}-1\right)=$ $\lambda_{1}-\lambda_{r}$ and hence $G$ is smooth. By $2.6, G \simeq G_{n, r}$ and this proves (5).

We finally determine the $k$-smooth groups of $k$-type $G_{k, k}$ or $(1, \ldots, 1)$, that is, with elementary abelian factors of order $p^{k}$.

THEOREM 2.8. Let $1<k \leq n$ and write $n=\lambda k+t$ where $\lambda \in \mathbb{N}$ and $0 \leq t<k$. The abelian p-group $G$ of order $p^{n}$ is $k$-smooth of $k$-type $(1, \ldots, 1)$ if and only if $G / \Omega_{\lambda}(G)$ is elementary abelian of order at most $p^{t}$, that is, $G$ has type $\left(\lambda_{1}, \ldots, \lambda_{r}\right)$ where $\lambda_{1} \leq \lambda+1$ and $\lambda_{t+1} \leq \lambda$.

ProOF. We use induction on $|G|$. Suppose first that $1=X_{0}<\cdots<X_{n}=G$ is a $k$-smooth chain with $X_{k}$ elementary abelian. If $t>0$, then $M:=X_{\lambda k}<G$ and, by Lemma $2.2, M$ is $k$-smooth of $k$-type $(1, \ldots, 1)$. The induction assumption yields that $M=\Omega_{\lambda}(M) \leq \Omega_{\lambda}(G)$. Since $n=\lambda k+t$, we have $|G / M|=p^{t}$ and $G / M \simeq X_{t} \leq X_{k}$ is elementary abelian. Thus $G / \Omega_{\lambda}(G)$ is elementary abelian of order at most $p^{t}$.

Now assume that $t=0$. Then for $\lambda=1$ we get that $G=X_{k}$ is elementary abelian, and if $\lambda \geq 2$, the induction assumption implies that $X_{n-k}=X_{(\lambda-1) k}$ has exponent at most $p^{\lambda-1}$. Since $G / X_{n-k} \simeq X_{k}$ is elementary abelian, it follows that $G=\Omega_{\lambda}(G)$, as desired. 
It is clear that $G / \Omega_{\lambda}(G)$ is elementary abelian of order at most $p^{t}$ if and only if $G$ has type $\left(\lambda_{1}, \ldots, \lambda_{r}\right)$ with $\lambda_{1} \leq \lambda+1$ and $\lambda_{t+1} \leq \lambda$. So suppose, conversely, that $G=A_{1} \times \cdots \times A_{r}$ with cyclic groups $A_{i}$ of order $p^{\lambda_{i}}$ where $\lambda+1 \geq \lambda_{1} \geq$ $\lambda_{2} \geq \cdots \geq \lambda_{r} \geq 1$ and $\lambda_{t+1} \leq \lambda$. Then $n=\sum_{i=1}^{r} \lambda_{i} \leq r \lambda+t$ and hence $k \leq r$. Thus $N:=\Omega\left(A_{1}\right) \times \cdots \times \Omega\left(A_{k}\right)$ is elementary abelian of order $p^{k}$ and $|G / N|=p^{m}$ where $m=(\lambda-1) k+t$. We write $\bar{U}=U N / N$ for every $U \leq G$ and show next that there exists a $k$-smooth chain

$$
1=X_{k} / N<X_{k+1} / N<\cdots<X_{n} / N=\bar{G} \quad \text { of } l \text {-type }(1, \ldots, 1),
$$

where $l=\min (m, k)$. This is clear if $m<k$; for then $\lambda=1$ and $\left|\bar{A}_{i}\right|=p$ for all $i$ since $t<k$. Thus $\bar{G}$ is elementary abelian in this case and has the desired chain. So suppose that $m \geq k$. Then (6) follows from the induction assumption if $\bar{G} / \Omega_{\lambda-1}(\bar{G})$ is elementary abelian of order at most $p^{t}$. Since $t<k,\left|\bar{A}_{i}\right| \leq p^{\lambda}$ for all $i$ and hence $\bar{G} / \Omega_{\lambda-1}(\bar{G})$ clearly is elementary abelian. So suppose, for a contradiction, that $\left|\bar{G} / \Omega_{\lambda-1}(\bar{G})\right| \geq p^{t+1}$. Assume that $d$ of the $A_{i}$ have order $p^{\lambda+1}$. Then $i \leq t$ for these $A_{i}$ and hence $d \leq t$. Since $\left|\bar{A}_{i}\right| \leq p^{\lambda-1}$ for $t<i \leq k$, there exist at least $t+1-d$ indices $i$ for which $k<i$ and $p^{\lambda}=\left|\bar{A}_{i}\right|=\left|A_{i}\right|$. Thus $\lambda_{k+t+1-d}=\lambda$ and it follows that $\left|A_{1} \times \cdots \times A_{k+t+1-d}\right|=p^{w}$ where

$$
\begin{aligned}
w & =d(\lambda+1)+(k+t+1-2 d) \lambda \\
& =k \lambda+(t-d) \lambda+d+\lambda \\
& \geq k \lambda+t+1=n+1
\end{aligned}
$$

since $\lambda \geq 1$. This contradiction proves (6).

We finally put $X_{0}=1$ and inductively define subgroups $X_{i}$ of $N(i=1, \ldots, k-1)$ such that $X_{i-1}<X_{i}$ and $\left|X_{i}\right|=p^{i}$ for all $i$, and $X_{i+k} / X_{i}$ is elementary abelian for all $i$ with $i+k \leq n$. Then all factor groups $X_{u} / X_{v}$ of order at most $p^{k}$ in the chain $1=X_{0}<\cdots<X_{n}=G$ will be elementary abelian and therefore the chain will be $k$-smooth of $k$-type $(1, \ldots, 1)$. So suppose that subgroups $X_{0}, \ldots, X_{i}$ with these properties have been defined and that $i<k-1$. Then if $i+k \geq n$, any subgroup $X_{i+1}$ of order $p^{i+1}$ of $N$ containing $X_{i}$ will do the job. If $i+k<n$, then since $X_{i+k} / X_{i}$ is elementary abelian, $X_{i+k+1} / X_{i}$ is of type $(2,1, \ldots, 1)$ or $(1, \ldots, 1)$. In the first case we take $X_{i+1} / X_{i}=\Phi\left(X_{i+k+1} / X_{i}\right)$; in the second case we let $X_{i+1} / X_{i}$ be any subgroup of order $p$ of $N / X_{i}$. Since $X_{i+k+1} / N$ is elementary abelian, in both cases $X_{i+1} \leq N$ and $X_{i+1+k} / X_{i+1}$ is elementary abelian.

We shall need two simple properties of smooth groups.

LEMMA 2.9. Let $1 \leq r<n$ and suppose that $1=X_{0}<\cdots<X_{n}=G$ is $a$ smooth chain in $G=G_{n, r}$; let $p^{e}$ be the exponent of $G$. Then there exist $g \in G$ and 
$K=S \times T \leq G$ such that $o(g)=p^{e}, S$ is of type $(e, \ldots, e)$ or $S=1, T$ is of type $(e-1, \ldots, e-1)$ or $T=1$, and satisfying $G=\langle g\rangle \times K, X_{n-1}=\left\langle g^{p}\right\rangle \times K, X_{n-r}=$ $\left\langle g^{p}\right\rangle \times K^{p}, X_{n-r-1}=\left\langle g^{p^{2}}\right\rangle \times K^{p}$.

Proof. Since $r<n$, we have $e>1$ and $G=R \times T$ where $R$ is of type $(e, \ldots, e)$ and $T$ is of type $(e-1, \ldots, e-1)$ or $T=1$. So $T \leq \Omega_{e-1}(G) \leq X_{n-1}$, by Lemma 2.4, and hence $X_{n-1}=\left(R \cap X_{n-1}\right) \times T$. Since $R \cap X_{n-1}$ is a maximal subgroup of $R$, we have $R \cap X_{n-1}=\langle u\rangle \times S$ where $o(u)=p^{e-1}$ and $S$ is of type $(e, \ldots, e)$ or $S=1$. Since $R$ is homocyclic, there exists $g \in R$ such that $g^{p}=u$. Then $R=\langle g\rangle \times S$ and hence $G=\langle g\rangle \times K$ and $X_{n-1}=\left\langle g^{p}\right\rangle \times K$ where $K=S \times T$. By 2.4, $X_{n-r}=G^{p}=\left\langle g^{p}\right\rangle \times K^{p}$ and $X_{n-r-1}=\left(X_{n-1}\right)^{p}=\left\langle g^{p^{2}}\right\rangle \times K^{p}$.

LEMMA 2.10. Suppose that $G \simeq H$ and that $1=X_{0}<\cdots<X_{n}=G$ and $1=$ $Y_{0}<\cdots<Y_{n}=H$ are smooth chains. Then every isomorphism $\alpha: X_{n-1} \rightarrow Y_{n-1}$ satisfying $X_{i}^{\alpha}=Y_{i}$ for $i=1, \ldots, n-2$ can be extended to an isomorphism of $G$ to $H$.

PROOF. If $G$ and $H$ are elementary abelian, it is clear that every isomorphism $\alpha: X_{n-1} \rightarrow Y_{n-1}$ can be extended. So we may assume that $G$ is not elementary abelian and by Corollary 2.6, $G \simeq G_{n, r}$ for some $r$ satisfying $1 \leq r<n$. Let $g, S, T$ be as in Lemma 2.9; since also $H \simeq G_{n, r}$, there exist $h, U, V$ having the corresponding properties with respect to the chain $1=Y_{0}<\cdots<Y_{n}=H$.

Now $X_{n-1}^{\alpha}=Y_{n-1}$ implies that $\left(g^{p}\right)^{\alpha}=h^{i p} u v$ with $i \in \mathbb{Z}, u \in U, v \in V$. By Lemma 2.4 there exists $j \in \mathbb{N}$ such that $X_{j}=G^{p^{e-1}}$ and $Y_{j}=H^{p^{c-1}}$. Since $X_{j}^{\alpha}=Y_{j}$, it follows that $H^{p^{e-1}}=\left\langle h^{p^{e-1}}\right\rangle \times \Omega(U)$ contains $\left(\left(g^{p}\right)^{p^{e-2}}\right)^{\alpha}=h^{i p^{e-1}} u^{p^{e-2}} v^{p^{e-2}}$; this yields $u^{p^{e-1}}=1=v^{p^{e-2}}$. Since $U$ and $V$ are trivial or homocyclic of exponent $p^{e}$ and $p^{e-1}$, respectively, there exist $x \in U, y \in V$ such that $x^{p}=u$ and $y^{p}=v$. Thus $\left(g^{p}\right)^{\alpha}=h^{i p} u v=\left(h^{i} x y\right)^{p}$ and so $o\left(h^{i} x y\right)=p^{e}$. For $K=S \times T$ we have $X_{n-1}^{\alpha}=\left\langle g^{p}\right\rangle^{\alpha} \times K^{\alpha}$ and hence $\left\langle h^{i} x y\right\rangle \cap K^{\alpha}=\left\langle g^{p}\right\rangle^{\alpha} \cap K^{\alpha}=1$. It follows that $H=\left\langle h^{i} x y\right\rangle \times K^{\alpha}$ and thus there exists an isomorphism $\beta: G \rightarrow H$ satisfying $g^{\beta}=h^{i} x y$ and $w^{\beta}=w^{\alpha}$ for $w \in K$. Since $\left(g^{p}\right)^{\beta}=\left(g^{\beta}\right)^{p}=\left(h^{i} x y\right)^{p}=\left(g^{p}\right)^{\alpha}, \beta$ is an extension of $\alpha$.

\section{Canonical $L$-embeddings and bases}

In this section we shall prove that if $G$ and $H$ have the structure given in Theorem $C$, then there exists an $L$-embedding of $G$ in $H$. For this we use Barnes' methods and therefore need some of the concepts introduced by him. For the convenience of the reader we recall them briefly. 
Let $G$ and $H$ be abelian $p$-groups of the same order and let $X_{1}, Y_{1}, X_{2}, Y_{2}$ be subgroups of $G$ such that $X_{2}=X_{1} \cup Y_{2}$ and $Y_{1}=X_{1} \cap Y_{2}$. Then the mappings

$$
\begin{array}{lll}
\sigma_{1}: X_{1} / Y_{1} \rightarrow X_{2} / Y_{2} ; & x Y_{1} \mapsto x Y_{2} & \left(x \in X_{1}\right) \\
\sigma_{2}: X_{2} / Y_{2} \rightarrow X_{1} / Y_{1} ; & x Y_{2} \mapsto x Y_{2} \cap Y_{1} & \left(x \in X_{2}\right)
\end{array}
$$

are mutually inverse isomorphisms. Such isomorphisms are called projectivities. If $\varphi: L(G) \rightarrow L(H)$ is an $L$-embedding, then by $(1), X_{2}^{\varphi}=X_{1}^{\varphi} \cup Y_{2}^{\varphi}$ and $Y_{1}^{\varphi}=X_{1}^{\varphi} \cap Y_{2}^{\varphi}$ so that there exist the corresponding projectivities

$$
\bar{\sigma}_{1}: X_{1}^{\varphi} / Y_{1}^{\varphi} \rightarrow X_{2}^{\varphi} / Y_{2}^{\varphi} \quad \text { and } \quad \bar{\sigma}_{2}: X_{2}^{\varphi} / Y_{2}^{\varphi} \rightarrow X_{1}^{\varphi} / Y_{1}^{\varphi}
$$

in the group $H$. For a closed chain

$$
c: X / Y=X_{1} / Y_{1} \stackrel{\sigma_{1}}{\longrightarrow} X_{2} / Y_{2} \stackrel{\sigma_{2}}{\longrightarrow} \cdots \stackrel{\sigma_{n-1}}{\longrightarrow} X_{n} / Y_{n}=X / Y
$$

of projectivities in $G$, the composition $\alpha(c):=\sigma_{1} \ldots \sigma_{n-1}$ is an automorphism of $X / Y$ and

$$
\bar{c}: X^{\varphi} / Y^{\varphi}=X_{1}^{\varphi} / Y_{1}^{\varphi} \stackrel{\bar{\sigma}_{1}}{\longrightarrow} X_{2}^{\varphi} / Y_{2}^{\varphi} \stackrel{\bar{\sigma}_{2}}{\longrightarrow} \cdots \stackrel{\bar{\sigma}_{n-1}}{\longrightarrow} X_{n}^{\varphi} / Y_{n}^{\varphi}=X^{\varphi} / Y^{\varphi}
$$

is a closed chain of projectivities in $H$. So if $X / Y$ is cyclic of order $p$ - we call $X / Y$ a prime interval in this case - there exist integers $r(c)$ and $r(\bar{c})$ prime to $p$ such that $x^{\alpha(c)}=x^{r(c)}$ for all $x \in X / Y$ and $y^{\alpha(c)}=y^{r(c)}$ for all $y \in X^{\varphi} / Y^{\varphi}$.

Definition 3.1. The $L$-embedding $\varphi: L(G) \rightarrow L(H)$ is called canonical if $r(c) \equiv r(\bar{c})(\bmod p)$ for all closed chains $c$ of projectivities on prime intervals in $G$.

Barnes uses the term '1-canonical' instead of 'canonical' since he considers, more generally, $L$-embeddings having similar properties with respect to cyclic intervals of order $p^{n}(n \in \mathbb{N})$ which he then terms ' $n$-canonical'. He shows [1, Theorem 3.1 and 3.3] that if $C$ and $\bar{C}$ are cyclic groups of order $p$ and $\varphi$ is a canonical $L$-embedding of $G$ in $H$, then $\varphi$ can be extended to a canonical $L$-embedding of $G \times C$ in $H \times \bar{C}$. Thus to prove Theorem $C$, it will suffice to show that $A \times B$ has a canonical $L$-embedding in $U \times V$; this can then be extended to $A \times B \times C_{3} \times \cdots \times C_{r}$.

Again let $\varphi: L(G) \rightarrow L(H)$ be an $L$-embedding. We consider the set $\mathbb{A}$ of all pairs $(x, y)$ where $x$ is a generator of a prime interval $X / Y$ of $G$ and $y$ is a generator of $X^{\varphi} / Y^{\varphi}$. A subset of $\mathfrak{a}$ is called a basis of $\varphi$ if for every prime interval $X / Y$ in $G$ there is a unique pair $(x, y) \in$ such that $X / Y=\langle x\rangle$ and $X^{\varphi} / Y^{\varphi}=\langle y\rangle$.

DEFINITION 3.2. Let 3 be a basis of $\varphi$. 
(a) A projectivity $\sigma: X_{1} / Y_{1} \rightarrow X_{2} / Y_{2}$ between prime intervals in $G$ is called regular with respect to if there exists $r \in \mathbb{Z}$ such that $x_{1}^{\sigma}=x_{2}^{r}$ and $y_{1}^{\sigma}=y_{2}^{r}$, where $\left(x_{i}, y_{i}\right) \in$ satisfy $X_{i} / Y_{i}=\left\langle x_{i}\right\rangle$ and $X_{i}^{\varphi} / Y_{i}^{\varphi}=\left\langle y_{i}\right\rangle(i=1,2)$.

(b) is called canonical if every projectivity between prime intervals in $G$ is regular with respect to $\mathrm{B}$.

It was noted by Barnes [1, p. 21] that an $L$-embedding is canonical if and only if it has a canonical basis. We need some simple properties of canonical bases.

LEMMA 3.3. Suppose that is a canonical basis of $\varphi$, let $X / Y$ be a prime interval in $G$ and let $(x, \bar{x}) \in \mathbf{3}$ be such that $X / Y=\langle x\rangle$. If $Z, W \leq G$ are such that $X \cap Z=Y$ and $X \cup Z=W$, then there exists $i \in \mathbb{Z}, i \not \equiv 0(\bmod p)$ such that $\left(x^{i} Z, \bar{x}^{i} Z^{\varphi}\right) \in \mathbf{Z}$.

Proof. Consider the projectivity $\sigma: X / Y \rightarrow W / Z$ and let $(w, \bar{w}) \in$ be such that $W / Z=\langle w\rangle$. Since is canonical, there exists $r \in \mathbb{Z}$ such that $x Z=x^{\sigma}=w^{r}$ and $\bar{x} Z^{\varphi}=\bar{x}^{\bar{\sigma}}=\bar{w}^{r}$. Thus $w=(x Z)^{i}$ and $\bar{w}=\left(\bar{x} Z^{\varphi}\right)^{i}$ for some $i \not \equiv 0(\bmod p)$.

LEMMA 3.4. Suppose that $\varphi$ is induced by an isomorphism $\varrho: G \rightarrow H$. Then $\varphi$ is canonical and we obtain a canonical basis of $\varphi$ if we choose for every prime interval in $G$ a generator $x$ and put the pair $\left(x, x^{\varrho}\right)$ in $\mathbf{0}$.

PROOF. If $\sigma: X / Y \rightarrow W / Z$ is a projectivity between prime intervals and $x, w$ are the chosen generators of $X / Y$ and $W / Z$, respectively, then the definition of $\bar{\sigma}$ implies that $\left(x^{\varrho}\right)^{\bar{\sigma}}=\left(x^{\sigma}\right)^{\varrho}$. So if $x^{\sigma}=w^{r}$, it follows that $\left(x^{Q}\right)^{\bar{\sigma}}=\left(w^{r}\right)^{\varrho}=\left(w^{\varrho}\right)^{r}$. Thus $\sigma$ is regular.

Conversely, we need that every canonical basis is of this type if $G$ and $H$ are elementary abelian (of order $p^{2}$ ).

LEMMA 3.5. Let $G$ and $H$ be elementary abelian of order $p^{2}$ and let $\varphi$ be a canonical $L$-embedding of $G$ in $H$ with canonical basis 3 . Suppose that $a, b \in G$ are such that $\langle a\rangle \neq\langle b\rangle$ and that contains the pairs $(a, x)$ and $(b, y)$. Then $\varphi$ is induced by the isomorphism $\varrho: G \rightarrow H ; a^{i} b^{j} \mapsto x^{i} y^{j}$, and for every pair $(c, z) \in$ we have $z=c^{e}$.

Proof. We clearly have $G=\langle a\rangle \times\langle b\rangle,\langle a\rangle^{\varphi}=\langle x\rangle$ and $\langle b\rangle^{\varphi}=\langle y\rangle$, so that $H=\langle x\rangle \times\langle y\rangle$. Suppose that $\left\langle a b^{k}\right\rangle^{\varphi}=\left\langle x y^{m}\right\rangle$ and let $\left(\left(a b^{k}\right)^{r},\left(x y^{m}\right)^{s}\right) \in$ where $1 \leq k, m, r, s \leq p-1$. By 3.3 there exist $i, j \in \mathbb{Z}$ such that $a^{r} b^{k r}\langle b\rangle=a^{i}\langle b\rangle$ and $x^{s} y^{m s}\langle y\rangle=x^{i}\langle y\rangle$ and also $a^{r} b^{k r}\langle a\rangle=b^{j}\langle a\rangle$ and $x^{s} y^{m s}\langle x\rangle=y^{j}\langle x\rangle$. It follows that $r \equiv i \equiv s(\bmod p)$ and $k r \equiv j \equiv m s(\bmod p)$ and hence $r=s$ and $k=m$. Thus $\varphi$ is induced by $\varrho$ and the pairs $(c, z) \in$ belonging to prime intervals $C / 1$ satisfy $z=c^{\ell}$. By 3.3, this then also holds for the prime intervals $G / C$. 
If $M \leq G$ and $\varphi$ is an $L$-embedding of $G$ in $H$, then the restriction of $\varphi$ to $L(M)$ is an $L$-embedding of $M$ in $M^{\varphi}$ which we call $\varphi_{M}$. If $\mathfrak{B}$ is a basis of $\varphi$, we let $\mathbf{B}_{M}$ be the set of pairs $(x, y) \in$ belonging to prime intervals $X / Y$ with $X \leq M$; clearly, $\mathfrak{x}_{M}$ is a basis of $\varphi_{M}$.

We want to construct canonical $L$-embeddings of groups of type $(n, k)$. This we shall do by induction, extending $L$-embeddings of smaller groups in the following obvious way; of course, the lemma holds more generally.

LEMMA 3.6. Suppose that $|G: F|=p^{2}$, where $F=\Phi(G)$; let $K \leq H$ and $\mu$ be an $L$-embedding of $G / F$ in $H / K$ with basis $\mathbf{3}_{\mu}$.

Let be the set of maximal subgroups of $G$ and for every $M \in$ let $\varphi(M)$ be an $L$-embedding of $M$ in $\bar{M}$ with basis $(M)$ where $\bar{M} / K=(M / F)^{\mu}$. Assume further that $\varphi(M)_{F}=\varphi(N)_{F}$ and $\mathbf{3}(M)_{F}=\mathbf{M}(N)_{F}$ for all $M, N \in \mathfrak{A t}$.

(a) Then $\psi: L(G) \rightarrow L(H)$, defined by $G^{\psi}=H$ and $X^{\psi}=X^{\varphi(M)}$ if $X \leq M \in$ Af, is an L-embedding of $G$ in $H$.

(b) If for every $M \in \mathfrak{A}$, the bases $\mathrm{H}_{\mu}$ and $(M)$ contain the same pair belonging to $M / F$, then the union $\mathbb{C}$ of ${ }_{\mu}$ and all the $\mathbf{B}(M)$ is a basis of $\psi$. If, in addition, $\mathbf{B}_{\mu}$ and all the $\mathbf{3}(M)$ are canonical, then so is $\mathbb{C}$.

PROOF. (a) Since the $L$-embeddings $\varphi(M)$ and $\varphi(N)$ coincide on $F=M \cap N$ for different $M, N \in$ the map $\psi$ is well-defined and injective. By definition, we have to show that if $X$ covers $Y$, then $X^{\psi}$ covers $Y^{\psi}$. This is clear if $X \leq M \in$, since in that case $\psi$ coincides with $\varphi(M)$ on $X$ and $Y$; and if $X=G$, then $Y^{\psi}=Y^{\varphi(Y)}$ is a maximal subgroup of $H$, since $Y^{\varphi(Y)} / K=(Y / K)^{\mu}$.

(b) Our assumptions imply that for a given prime interval $X / Y$, all the bases $\boldsymbol{B}(M)$ and $\mathbf{b}_{\mu}$ which contain a pair belonging to $X / Y$ contain the same pair. Thus the union $\mathbb{I}$ is a basis of $\psi$. If $\mathbf{B}_{\mu}$ and all the $(M)$ are canonical, then by [1, Lemma 4.1], so is I.

The main difficulty in extending a given $L$-embedding of a maximal subgroup to a canonical $L$-embedding of $G$ in $H=U \times V$ is that this extension can be done rather arbitrarily if $V$ is elementary abelian, has to be constructed suitably if $|V / \Omega(V)|=p$, but is uniquely determined if $|V / \Omega(V)| \geq p^{2}$. Fortunately, we only have to study this in the case $n=k$.

Lemma 3.7. Let $G=A \times B$ with cyclic groups $A$ and $B$ of order $p^{n}$, let $\varphi$ be an L-embedding of $G$ in $H$ and suppose that $H$ is not elementary abelian. Write $F=\Phi(G), U=A^{\varphi}, V=B^{\varphi} ;$ for $i=0, \ldots, n$, let $A_{i} \leq A$ and $B_{i} \leq B$ with $\left|A_{i}\right|=p^{i}=\left|B_{i}\right|, U_{i}=A_{i}^{\varphi}$ and $V_{i}=B_{i}^{\varphi}$. Finally, suppose that is a basis of $\varphi$ and that $|\Omega(U)|=p^{r}$. 
(a) There exist generators $a$ of $A$ and $b$ of $B$, elements $u \in U, v \in V$, and integers $i, j, s, t \in\{1, \ldots, p-1\}$ such that $\mathbf{B}$ contains the pairs $\left(a A_{n-1}, u U_{n-1}\right)$, $\left(a^{i p^{r}} A_{n-r-1}, u^{i s p} U_{n-r-1}\right),\left(b B_{n-1}, v V_{n-1}\right)$ and $\left(b^{j p^{r}} B_{n-r-1}, v^{j t p} V_{n-r-1}\right)$.

(b) If $\mathfrak{\mathbf { B }}_{F}$ is a canonical basis of $\varphi_{F}$ and $a, b, u, v, s, t$ are as in (a), then $\varphi$ is induced on $G / F$ by the isomorphism $\sigma: G / F \rightarrow H / F^{\varphi}$ given by $(a F)^{\sigma}=u^{s} F^{\varphi}$ and $(b F)^{\sigma}=v^{t} F^{\varphi}$.

(c) If is a canonical basis of $\varphi$, then, in addition, $s=t$.

PROOF. (a) By 2.3, $1=U_{0}<\cdots<U_{n}=U$ and $1=V_{0}<\cdots<V_{n}=V$ are smooth chains, $H=U \times V$ and $U \simeq V$. Since $H$ is not elementary abelian, it follows that neither $U$ nor $V$ is; thus $r<n$. By 2.6, $U \simeq G_{n, r}$ and by 2.9 there exist $u \in U$ and $K \leq U$ such that

$$
U=\langle u\rangle \times K, U_{n-1}=\left\langle u^{p}\right\rangle \times K, U_{n-r}=\left\langle u^{p}\right\rangle \times K^{p}, U_{n-r-1}=\left\langle u^{p^{2}}\right\rangle \times K^{p} .
$$

Thus $U / U_{n-1}=\left\langle u U_{n-1}\right\rangle$ and hence $\mathbf{B}$ contains an element of the form $\left(a A_{n-1}, u^{k} U_{n-1}\right)$ where $A=\langle a\rangle$ and $k \not \equiv 0(\bmod p)$. So if we replace $u$ by $u^{k}$, we still have (7) and $\left(a A_{n-1}, u U_{n-1}\right) \in$ B. Since $A_{n-r} / A_{n-r-1}=\left\langle a^{p^{r}} A_{n-r-1}\right\rangle$ and $U_{n-r} / U_{n-r-1}=$ $\left\langle u^{p} U_{n-r-1}\right\rangle$, there exist $i, s \in\{1, \ldots, p-1\}$ such that contains $\left(a^{i p^{r}} A_{n-r-1}\right.$, $\left.u^{i s p} U_{n-r-1}\right)$. In the same way we get $b, v, j, t$ with the desired properties.

(b) Let $W=A_{n-r} \times B_{n-r}, R=A_{n-r} \times B_{n-r-1}, S=A_{n-r-1} \times B_{n-r}$, and $T=$ $A_{n-r-1} \times B_{n-r-1}$. Then $W / T=R / T \times S / T$ is elementary abelian of order $p^{2}$. Since $R / T$ is projective to $A_{n-r} / A_{n-r-1}$ and $B_{F}$ is canonical, by 3.3 there exists $v \in \mathbb{Z}$ such that $\mathbf{B}_{F}$ contains $\left(a^{i p^{r} v} T, u^{i s p v} T^{\varphi}\right)$; similarly, there exists $\mu \in \mathbb{Z}$ such that $\left(b^{j p^{r} \mu} T, v^{j t p \mu} T^{\varphi}\right) \in \mathbf{B}_{F}$ belongs to the interval $S / T$. By $3.5, \varphi$ is induced on $W / T$ by the isomorphism $\varrho: W / T \rightarrow W^{\varphi} / T^{\varphi}$ satisfying $\left(a^{i p^{r} v} T\right)^{\varrho}=u^{i s p v} T^{\varphi}$ and $\left(b^{j p^{r} \mu} T\right)^{\varrho}=v^{j t p \mu} T^{\varphi}$; it follows that

$$
\left(a^{p^{r}} T\right)^{\varrho}=u^{s p} T^{\varphi} \quad \text { and } \quad\left(b^{p^{r}} T\right)^{\varrho}=v^{t p} T^{\varphi} .
$$

For $\lambda \in\{1, \ldots, p-1\}$ let $D=D_{\lambda}=\left\langle a b^{\lambda}\right\rangle$. This is a diagonal in $G=A \times B$; by (1), $D^{\varphi}$ is a diagonal in $H=U \times V$; that is, $D^{\varphi}=D(\alpha)=\left\{x x^{\alpha} \mid x \in U\right\}$ for some isomorphism $\alpha: U \rightarrow V$ (see [4, Theorem 1.6.2]). Since $D \cap F=\left\langle a^{p} b^{\lambda p}\right\rangle$ is a diagonal in $F=A_{n-1} \times B_{n-1},(D \cap F)^{\varphi}=D^{\varphi} \cap F^{\varphi}$ is a diagonal in $F^{\varphi}=U_{n-1} \times V_{n-1}$ and hence $U_{n-1}^{\alpha}=V_{n-1}$. We have $U / U_{n-1}=\left\langle u U_{n-1}\right\rangle$ and $V / V_{n-1}=\left\langle v V_{n-1}\right\rangle$; thus there exists $d=d_{\lambda} \in\{1, \ldots, p-1\}$ such that $u^{\alpha}=v^{d} w$ with $w \in V_{n-1}$. Since $(D F)^{\varphi}=D^{\varphi} F^{\varphi}$, it follows that

$$
\left(\left\langle a b^{\lambda}\right\rangle F\right)^{\varphi}=\left\langle u v^{d_{\lambda}}\right\rangle F^{\varphi} .
$$

Now $(D \cap W) T / T=\left\langle a^{p^{r}} b^{\lambda p^{r}}\right\rangle T / T$ is a diagonal in $W / T$ which, by (8), is mapped to $\left\langle u^{s p} v^{\lambda t p}\right\rangle T^{\varphi} / T^{\varphi}$ by $\varphi$. On the other hand, $(D \cap W) \cup T$ is mapped to

$$
\left(D^{\varphi} \cap W^{\varphi}\right) \cup T^{\varphi}=\left\{x x^{\alpha} \mid x \in U_{n-r}\right\} T^{\varphi}=\left\langle u^{p} v^{d p}\right\rangle T^{\varphi}=\left\langle u^{s p} v^{s d p}\right\rangle T^{\varphi}
$$


since $\left(\boldsymbol{u}^{p}\right)^{\alpha}=v^{d p} w^{p}$ and $w^{p} \in V_{n-1}^{p}=V_{n-r-1} \leq T^{\varphi}$; see (7). It follows that $\lambda t \equiv s d(\bmod p)$. Now $d=d_{\lambda}$ and (9) yields that for $\lambda=1, \ldots, p-1$,

$$
\left(\left\langle a b^{\lambda}\right\rangle F\right)^{\varphi}=\left\langle u^{s} v^{s d_{\lambda}}\right\rangle F^{\varphi}=\left\langle u^{s} v^{\lambda t}\right\rangle F^{\varphi} ;
$$

that is, $\varphi$ is induced on $G / F$ by the isomorphism $\sigma$ satisfying $(a F)^{\sigma}=u^{s} F^{\varphi}$ and $(b F)^{\sigma}=v^{t} F^{\varphi}$.

(c) Since is a canonical basis containing $\left(a A_{n-1}, u U_{n-1}\right)$ and $A F / F$ is projective to $A / A_{n-1}$, Lemma 3.3 implies that there exists $v \in \mathbb{Z}$ such that $\left(a^{v} F, u^{v} F^{\varphi}\right) \in \mathbf{B}$; similarly, $\left(b^{\mu} F, v^{\mu} F^{\varphi}\right) \in \mathbb{B}$ for some $\mu \in \mathbb{Z}$. By $3.5, \varphi$ is induced on $G / F$ by the isomorphism $\tau: G / F \rightarrow H / F^{\varphi}$ satisfying $(a F)^{\tau}=u F^{\varphi}$ and $(b F)^{\tau}=v F^{\varphi}$. Now $\sigma$ and $\tau$ induce the same lattice isomorphism of $G / F$ and hence $\sigma \tau^{-1}$ is a power automorphism. It follows that $s=t$.

LEMMA 3.8. Let $G$ be of type $(n, n)$, suppose that $\varphi$ is an L-embedding of $G$ in $H$ and that $|H / \Omega(H)|>p^{2}$. Let $F=\Phi(G)$ and be the set maximal subroups of $G$ and assume further that for every $M \in$ the induced L-embedding $\varphi_{M}$ is canonical and has a canonical basis $\mathbf{B}(M)$ such that $(M)_{F}=\boldsymbol{B}(N)_{F}$ for all $N \in$ ft. Then $\varphi$ is canonical and has a canonical basis $\mathbf{B}_{\text {such that }} \mathbf{B}_{M}=\mathfrak{B}(M)$ for all $M \in$.

PROOF. We show first that the lemma is an easy consequence of the following.

(10) For every two different maximal subgroups $M$ and $N$ of $G$ there exists an isomorphism $\sigma=\sigma_{M N}: G / F \rightarrow H / F^{\varphi}$ inducing $\varphi$ there and satisfying: if $(x, \bar{x}) \in \mathbf{B}(M)$ and $(y, \bar{y}) \in(N)$ are such that $M / F=\langle x\rangle$ and $N / F=\langle y\rangle$, then $x^{\sigma}=\bar{x}$ and $y^{\sigma}=\bar{y}$.

So assume that (10) holds, take $M, N \in$ such that $M \neq N$ and put $\sigma=\sigma_{M N}$. If $M \neq L \in$ and $(z, \bar{z}) \in(L)$ is such that $L / F=\langle z\rangle$, then $\tau=\sigma_{M L}$ induces $\varphi$ on $G / F$ and satisfies $x^{\tau}=\bar{x}$ and $z^{\tau}=\bar{z}$. Then $\sigma$ and $\tau$ induce the same lattice isomorphism of $G / F$, so $\sigma \tau^{-1}$ is a power automorphism which is trivial since $x^{\sigma}=x^{\tau}$. Thus $\sigma=\tau$ and hence $\sigma$ satisfies for all $L \in \mathfrak{f A}$,

$$
z^{\sigma}=\bar{z} \quad \text { if } \quad(z, \bar{z}) \in(L) \text { is such that } L / F=\langle z\rangle .
$$

Let $\mu$ be the $L$-embedding of $G / F$ in $H / F^{\varphi}$ induced by $\varphi$, and hence by $\sigma$; let $\mathrm{H}_{\mu}$ be the canonical basis of $\mu$ constructed in 3.4 using the pairs $\left(z, z^{\sigma}\right) \in \mathbf{B}(L)$ for $L \in$ A Then $\mathbf{B}_{\mu}$ and the $\mathbf{B}(M)$ satisfy the assumptions of $3.6(\mathrm{~b})$ and hence their union is a canonical basis of $\varphi$.

It remains to prove (10). Let be a basis of $\varphi$ that contains every $(L), L \in$ such a basis exists by $3.6(\mathrm{~b})$.

Now let $M, N \in$ be such that $M \neq N$. Then $M$ and $N$ are of type $(n, n-1)$ and hence $M=\langle c\rangle \times\langle d\rangle$ and $N=\langle x\rangle \times\langle y\rangle$ where $o(c)=o(x)=p^{n}$ and 
$o(d)=o(y)=p^{n-1}$. Since $M \neq N$, we have $G=M N=\langle c, x\rangle F$ and hence $G=\langle c\rangle \times\langle x\rangle$; furthermore $M=\langle c\rangle \times(M \cap\langle x\rangle)=\langle c\rangle \times\left\langle x^{p}\right\rangle$ and, similarly, $N=\left\langle c^{p}\right\rangle \times\langle x\rangle$. So if we put $A=\langle c\rangle$ and $B=\langle x\rangle$, we have that $G=A \times B$ where $A$ and $B$ are cyclic of order $p^{n}$. Let $U=A^{\varphi}, V=B^{\varphi}$, and for $i=0, \ldots, n$, let $A_{i} \leq A$ and $B_{i} \leq B$ be such that $\left|A_{i}\right|=p^{i}=\left|B_{i}\right|, U_{i}=A_{i}^{\varphi}, V_{i}=B_{i}^{\varphi}$. Then

$$
M=A \times B_{n-1} \quad \text { and } \quad N=A_{n-1} \times B .
$$

By 2.3, $H=U \times V, 1=U_{0}<\cdots<U_{n}=U$ and $1=V_{0}<\cdots<V_{n}=V$ are smooth chains, and $U \simeq V$. Let $|\Omega(U)|=p^{r}$. Since $|H / \Omega(H)|>p^{2}, U_{n-1}$ is not elementary abelian; thus, by 2.4 ,

$$
\Omega(U)<U_{n-1} \text { and } r<n-1 .
$$

If we apply 3.7 to $G=A \times B$, we obtain generators $a$ of $A$ and $b$ of $B$, elements $u \in U$ and $v \in V$, and integers $i, j, s, t \in\{1, \ldots, p-1\}$ such that contains the pairs $\left(a A_{n-1}, u U_{n-1}\right),\left(a^{i p^{r}} A_{n-r-1}, u^{i s p} U_{n-r-1}\right),\left(b B_{n-1}, v V_{n-1}\right)$, and $\left(b^{j p^{r}} B_{n-r-1}, v^{j t p} V_{n-r-1}\right)$. By (b) of 3.7,

(14) $\varphi$ is induced on $G / F$ by the isomorphism $\sigma^{*}: G / F \rightarrow H / F^{\varphi}$ satisfying $(a F)^{\sigma^{*}}=u^{s} F^{\varphi}$ and $(b F)^{\sigma^{*}}=v^{t} F^{\varphi}$.

Now we apply 3.7 to $F=A_{n-1} \times B_{n-1}$. By (13), the assumptions of 3.7 hold with $n$ replaced by $n-1$ but with the same $r$ as before. Therefore there exist generators $a_{1}$ of $A_{n-1}$ and $b_{1}$ of $B_{n-1}$, elements $u_{1} \in U_{n-1}$ and $v_{1} \in V_{n-1}$, and integers $\mu, \nu, m$ such that $\mathbf{3}_{F}$, and hence also $\mathbf{3}$, contains the pairs $\left(a_{1} A_{n-2}, u_{1} U_{n-2}\right),\left(a_{1}^{\mu p^{\prime}} A_{n-r-2}, u_{1}^{\mu m p} U_{n-r-2}\right)$, $\left(b_{1} B_{n-2}, v_{1} V_{n-2}\right)$, and $\left(b_{1}^{v p^{r}} B_{n-r-2}, v_{1}^{v m p} V_{n-r-2}\right)$; note that $\mathbf{b}_{F}$ is canonical so that 3.7(c) yields the same power of $u_{1}^{\mu p}$ and $v_{1}^{v p}$ here.

Next we apply 3.7 to $M / A_{1}=A F / A_{1}=A / A_{1} \times B_{n-1} A_{1} / A_{1}$, the canonical $L$ embedding induced by $\varphi_{M}$ in $M / A_{1}$ and the induced basis $(M)^{*}$; since $U / U_{1} \simeq U_{n-1}$, the assumptions of 3.7 hold. For short, we write $\tilde{x}$ and $\tilde{X}$ for the images of elements $x$ or subgroups $X$ under the natural homomorphisms of $G$ to $G / A_{1}$ and of $H$ to $H / U_{1}$. By (13), $A_{1} \leq A_{n-r-1}$ and hence $3(M)^{*}$ contains the pairs $\left(\tilde{a} \tilde{A}_{n-1}, \tilde{u} \tilde{U}_{n-1}\right)$ and $\left(\tilde{a}^{i p^{r}} \tilde{A}_{n-r-1}, \tilde{u}^{i s p} \tilde{U}_{n-r-1}\right)$; by 3.3 there exist $\lambda, \varrho \in \mathbb{Z}$ such that $(M)^{*}$ contains $\left(\tilde{b}_{1}^{\lambda} \tilde{B}_{n-2}, \tilde{v}_{1}^{\lambda} \tilde{V}_{n-2}\right)$ and $\left(\tilde{b}_{1}^{v e p^{\prime}} \tilde{B}_{n-r-2}, \tilde{v}_{1}^{v m \varrho p} \tilde{V}_{n-r-2}\right)$. Since $\mathbf{B}(M)^{*}$ is canonical, (c) of 3.7 implies that $m=s$.

If we finally apply 3.7 to the symmetric situation in the factor group $N / B_{1}=A_{n-1} B_{1} / B_{1} \times B / B_{1}$, we obtain that $m=t$. It follows that $s=t$.

Let $\tau$ be the power automorphism of $H / F^{\varphi}$ mapping every element $x$ to $x^{w}$ where $s w \equiv 1(\bmod p)$ and let $\sigma=\sigma^{*} \tau$. Then by (14), $\varphi$ is induced by $\sigma$ on $G / F$ and $(a F)^{\sigma}=u F^{\varphi},(b F)^{\sigma}=v F^{\varphi}$. Since $(M)$ is canonical and $M / F$ is projective to $A / A_{n-1}$, by 3.3 there exists $k \in \mathbb{Z}$ such that $\left(a^{k} F, u^{k} F^{\varphi}\right) \in \mathbb{B}(M)$ and $M / F=\left\langle a^{k} F\right\rangle$; similarly, there exists $l \in \mathbb{Z}$ such that $\left(b^{l} F, v^{l} F^{\varphi}\right) \in \mathbf{b}(N)$ and $\left\langle b^{l} F\right\rangle=N / F$. Since 
$\left(a^{k} F\right)^{\sigma}=u^{k} F^{\varphi}$ and $\left(b^{l} F\right)^{\sigma}=v^{l} F^{\varphi}$, the isomorphism $\sigma$ satisfies (10). This proves (10) and the lemma.

We can now construct the desired extension.

LeMMA 3.9. Let $1 \leq k \leq n$ and $G=A \times B$ with cyclic groups $A$ of order $p^{n}$ and $B$ of order $p^{k}$; for $i=0, \ldots, n$, let $A_{i} \leq A$ be such that $\left|A_{i}\right|=p^{i}$. Let $H=U \times V$ be abelian, $|U|=p^{n},|V|=p^{k}$, and let $1=U_{0}<\cdots<U_{n}=U$ be a $k$-smooth chain with $U_{k} \simeq V$.

Let $\varphi$ be a canonical L-embedding of $G_{0}=A_{n-1} \times B$ in $H_{0}=U_{n-1} \times V$ such that $A_{i}^{\varphi}=U_{i}$ for $i=1, \ldots, n-1$ and $B^{\varphi}=V$; let be a canonical basis of $\varphi$ and suppose that $G / G_{0}=\langle w\rangle$ and $H / H_{0}=\langle\bar{w}\rangle$.

Then there exists an extension of $\varphi$ to a canonical L-embedding $\psi$ of $G$ in $H$ satisfying $A^{\psi}=U$; furthermore there exists a canonical basis $\mathbb{C}$ of $\psi$ containing and, if $V$ is elementary abelian, the pair $(w, \bar{w})$.

Proof. We use induction on $|G|$. If $n=k=1$, then $G$ and $H$ are elementary abelian of order $p^{2}$ and there exists an isomorphism $\varrho: G \rightarrow H$ satisfying $A^{\varrho}=$ $U, w^{\varrho}=\bar{w}$, and $b^{Q}=v$ if $=\{(b, v)\}$; by 3.4 , the $L$-embedding $\psi$ induced by $\rho$ is canonical and has a canonical basis $\mathbb{C}$ containing $(b, v)$ and $(w, \bar{w})$.

So suppose that $n+k \geq 3$ and that the assertion is true for groups of smaller order.

Case 1: $n>k$. Then $N:=A_{n-k} \neq 1$ and $G_{0}=\Omega_{n-1}(G)$; by 2.2 , the assumptions of the lemma hold for the $L$-embedding $\tilde{\varphi}$ with canonical basis $\tilde{j}$ induced by $\varphi$ and $\mathfrak{3}$, respectively, in $G_{0} / N$. The induction assumption yields an extension $\tilde{\psi}$ of $\tilde{\varphi}$ to a canonical $L$-embedding of $G / N$ in $H / N^{\varphi}$ such that $(A / N)^{\tilde{\psi}}=U / N^{\varphi}$ and a canonical basis $\tilde{\mathbb{T}}$ of $\tilde{\psi}$ that contains $\tilde{\text { and }}$, if $V N / N \simeq V$ is elementary abelian, the pair $(w, \bar{w})$.

Let $1 \leq Y<X \leq G$ such that $|X: Y|=p$ and suppose that $X \not G_{0}$. Then $X$ contains an element $x=a b$ of order $p^{n}$, where $a \in A$ and $b \in B$, and $x^{p^{k}}=a^{p^{k}}$ generates $N$; thus $N \leq X$. Furthermore, if $N \not \geq Y$, it would follow that $Y \leq G_{0}$; but then $X=N Y \leq G_{0}$, a contradiction. We have shown:

If $X / Y$ is a prime interval in $G$, then $X \leq G_{0}$ or $N \leq Y$.

This shows that the map $\psi: L(G) \rightarrow L(H)$ given by $X^{\psi}=X^{\varphi}$ for $X \leq G_{0}$ and $X^{\psi} / N^{\varphi}=(X / N)^{\tilde{\psi}}$ if $X \not G_{0}$ is well-defined and, clearly, injective. To prove that $\psi$ is an $L$-embedding, by definition, we have to show that if $X$ covers $Y$, then $X^{\psi}$ covers $Y^{\psi}$. But (15) and the fact that $\tilde{\psi}$ is induced by $\varphi$ on $G_{0} / N$ yield that if $X$ covers $Y$, then $\psi$ coincides on $X$ and $Y$ either with $\varphi$ or ith the map induced by $\tilde{\psi}$. In both cases, $X^{\psi}$ covers $Y^{\psi}$. Thus $\psi$ is an $L$-embedding satisfying $A^{\psi}=U$ and we show that

$$
\mathfrak{C}:=\mathfrak{B} \cup\left\{(x, \bar{x}) \in \tilde{\mathbb{C}} \mid\langle x\rangle=X / Y, X \notin G_{0}\right\}
$$


is a canonical basis of $\psi$; here, to simplify notation, we identify generators of $X / Y$ and of $(X / N) /(Y / N)$ if $N \leq Y<X$. Indeed, by (15), $\mathbb{C}$ is a basis of $\psi$. We have to show that every projectivity $\sigma: X_{1} / Y_{1} \rightarrow X_{2} / Y_{2}$ between prime intervals of $G$ is regular with respect to $\mathbb{C}$ and by $\left[1\right.$, p. 21], we may assume that $X_{1}$ is a maximal subgroup of $X_{2}$. If $X_{2} \leq G_{0}$, then coincides with on the intervals $X_{i} / Y_{i}$; and if $X_{2} \not \leq G_{0}$, then by (15), $N \leq X_{1} \cap Y_{2}=Y_{1}$ and $\mathbb{C}$ coincides with $\tilde{\mathbb{C}}$ on these intervals. In both cases, $\sigma$ is regular and $\mathbb{C}$ is a canonical basis having all the desired properties.

Case 2: $n=k$ and $V$ is elementary abelian. Then $H$ is elementary abelian; we let $F=\Phi(G)$ and choose an isomorphism $\varrho: G / F \rightarrow H / F^{\varphi}$ satisfying $(A F / F)^{\varrho}=$ $U F^{\varphi} / F^{\varphi}, w^{\varrho}=\bar{w}$, and $z^{\varrho}=\bar{z}$ if $(z, \bar{z}) \in \mathfrak{B}$ is such that $G_{0} / F=\langle z\rangle$. Since in an elementary abelian group every maximal chain is smooth, by induction, for every maximal subgroup $M \neq G_{0}$ of $G$ there exists an extension of $\varphi_{F}$ to a canonical $L$ embedding $\varphi(M)$ of $M$ in $\bar{M}$ where $\bar{M} / F^{\varphi}=(M / F)^{Q}$; and $A^{\varphi(M)}=U$ if $M=A F$. Furthermore there exists a canonical basis $(M)$ of $\varphi(M)$ containing $\mathfrak{B}_{F}$ and the pair $\left(x, x^{Q}\right)$ for some generator $x$ of $M / F$. If $\mu$ is the $L$-embedding of $G / F$ in $H / F^{\varphi}$ induced by $\varrho$, then by $3.4, \mu$ is canonical and has a canonical basis $\mathbf{B}_{\mu}$ containing all these $\left(x, x^{\rho}\right)$ and $(w, \bar{w})$. Now the $L$-embedding $\psi$ and its canonical basis constructed in Lemma 3.6 have the desired properties.

Case 3: $n=k$ and $V$ is not elementary abelian. Then $A=\langle a\rangle$ and $B=\langle b\rangle$ with $o(a)=o(b)=p^{n}$; furthermore, by assumption, $V \simeq U_{n}=U$ and hence both groups are smooth. For $i=0, \ldots, n$, let $B_{i} \leq B$ be such that $\left|B_{i}\right|=p^{i}$, and let $V_{i}=B_{i}^{\varphi}$. Again write $F=\Phi(G)=A_{n-1} \times B_{n-1}$ and let be the set maximal subgroups of $G$. The induction assumption applied to $A F=A \times B_{n-1} \in$ yields the following.

(16) There exists an extension of $\varphi_{F}$ to a canonical $L$-embedding $\varphi(A F)$ of $A F$ in $U F^{\varphi}$ satisfying $A^{\varphi(A F)}=U$; furthermore there exists a canonical basis $\mathfrak{b}(A F)$ of $\varphi(A F)$ containing 3 and, if $V_{n-1}$ is elementary abelian, a given pair $(x, \bar{x})$ satisfying $A F / F=\langle x\rangle$ and $U F^{\varphi} / F^{\varphi}=\langle\bar{x}\rangle$.

We now handle the $M \in$ with $A F \neq M \neq B F=G_{0}$. These have the form

$$
M^{(j)}=D^{(j)} F=D^{(j)} \times B_{n-1} \quad \text { for } \quad j=1, \ldots, p-1,
$$

where $D^{(j)}=\left\langle a b^{j}\right\rangle$. Let $j \in\{1, \ldots, p-1\}$ and write $M=M^{(j)}$ and $D=D^{(j)}$. Then $D \cap F=\left\langle a^{p} b^{j p}\right\rangle$ is a diagonal in $F$ and therefore by $(1),(D \cap F)^{\varphi}$ is a diagonal in $F^{\varphi}=U_{n-1} \times V_{n-1}$. By [4, Theorem 1.6.2],

$$
(D \cap F)^{\varphi}=D\left(U_{n-1}, \tau\right)=\left\{u u^{\tau} \mid u \in U_{n-1}\right\}
$$


where $\tau: U_{n-1} \rightarrow V_{n-1}$ is an isomorphism. For $i=1, \ldots, n-1$, let $D_{i}$ be the subgroup of order $p^{i}$ of $D$. Then $D_{i}=\left(A_{i} \cup B_{n-1}\right) \cap(D \cap F)$ and hence by (1),

$$
D_{i}^{\varphi}=\left(U_{i} \cup V_{n-1}\right) \cap D\left(U_{n-1}, \tau\right)=\left\{u u^{\tau} \mid u \in U_{i}\right\}=U_{i}^{\tau^{*}}
$$

where $\tau^{*}$ is the natural isomorphism $\tau^{*}: U_{n-1} \rightarrow D\left(U_{n-1}, \tau\right) ; u \mapsto u u^{\tau}$. Furthermore, $B_{i}=\left(A_{i} \cup(D \cap F)\right) \cap B_{n-1}$ and hence

$$
V_{i}=\left(U_{i} \cup D\left(U_{n-1}, \tau\right)\right) \cap V_{n-1}=U_{i}^{\tau} .
$$

Thus we have shown that for all $i=1, \ldots, n-1$,

$$
U_{i}^{\tau}=V_{i} \quad \text { and } \quad U_{i}^{\tau^{*}}=D_{i}^{\varphi} .
$$

By 2.3, the chain $1=V_{0}<\cdots<V_{n}=V$ is $(n-1)$-smooth and hence smooth since $|V|=p^{n}$. By (18) and 2.10, there is an extension of $\tau$ to an isomorphism $\bar{\tau}: U \rightarrow V$; let $\bar{D}=D(U, \bar{\tau})$. Then $\bar{D}$ is a diagonal in $H=U \times V$ and hence $\bar{M}:=\bar{D} F^{\varphi}=\bar{D} \times V_{n-1}$ is a maximal subgroup of $H$ containing $F^{\varphi}$. By (18), the natural isomorphism $\bar{\tau}^{*}: U \rightarrow \bar{D} ; u \mapsto u u^{\bar{\tau}}$ maps $U_{i}$ to $D_{i}^{\varphi}$ for $i=1, \ldots, n-1$ and therefore $1=D_{0}^{\varphi}<\cdots<D_{n-1}^{\varphi}<\bar{D}$ is a smooth chain. Since $\bar{D} \simeq U$ is not elementary abelian, 2.4 yields that

$$
\Omega(\bar{D}) \leq D_{n-1}^{\varphi}=(D \cap F)^{\varphi} .
$$

Furthermore we see that $M=D \times B_{n-1}, \bar{M}=\bar{D} \times V_{n-1}, \varphi_{F}, \mathbf{D}_{F}$ satisfy the assumptions of the lemma in place of $G, H, \varphi, \mathbf{n}$, and hence the induction assumption finally implies the following.

(20) There exist an extension of $\varphi_{F}$ to a canonical $L$-embedding $\varphi(M)$ of $M$ in $\bar{M}$ and a canonical basis $(M)$ of $\varphi(M)$ containing $M_{F}$ and, if $V_{n-1}$ is elementary abelian, a given pair $(x, \bar{x})$ satisfying $M / F=\langle x\rangle$ and $\bar{M} / F^{\varphi}=\langle\bar{x}\rangle$.

Now if $i, j \in\{1, \ldots, p-1\}$ with $i \neq j$, then $D^{(i)} \cap D^{(j)}=\left\langle a b^{i}\right\rangle \cap\left\langle a b^{j}\right\rangle=1$ and hence by $(1),\left(D^{(i)} \cap F\right)^{\varphi} \cap\left(D^{(j)} \cap F\right)^{\varphi}=1$. Thus by $(19), \overline{D^{(i)}}$ and $\overline{D^{(j)}}$ are diagonals of $H=U \times V$ intersecting trivially, and therefore they generate $H$; it follows that $\overline{M^{(i)}} \neq \overline{M^{(j)}}$. This shows that there is an $L$-embedding $\mu$ of $G / F$ in $H / F^{\varphi}$ satisfying $(A F / F)^{\mu}=U F^{\varphi} / F^{\varphi},\left(G_{0} / F\right)^{\mu}=H_{0} / F^{\varphi}$, and $\left(M^{(j)} / F\right)^{\mu}=\overline{M^{(j)}} / F^{\varphi}$ for $j=1, \ldots, p-1$. If we write $\varphi=\varphi\left(G_{0}\right)$ and $=0\left(G_{0}\right)$, then by (16) and (20), $\mu$ and the $L$-embeddings $\varphi(M)$ and bases $(M)(M \in$ ) satisfy the assumptions of Lemma 3.6. Let $\psi$ be the $L$-embedding of $G$ in $H$ constructed there; then $\psi_{M}=\varphi(M)$ for every $M \in$ and $\psi$ induces $\mu$ on $G / F$.

If $V_{n-1}$ is not elementary abelian, then by $2.4, \Omega(V)<V_{n-1}$ and hence $|H / \Omega(H)|$ $>p^{2}$. By $3.8, \psi$ is canonical and has a canonical basis $\mathbb{}$ such that $\mathbb{\leftarrow}_{M}=\mathbf{m}(M)$ for all $M \in$ In particular, $A^{\psi}=U$ and $\mathbb{C}$ contains $\mathbb{H}$, as desired. 
So assume, finally, that $V_{n-1}$ is elementary abelian. Since $H$ is not elementary abelian, (b) of 3.7 implies that $\psi$, and hence also $\mu$, is induced on $G / F$ by an

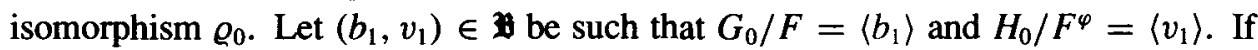
we add a suitable power automorphism of $H / F^{\varphi}$ to $\varrho_{0}$, we obtain an isomorphism $\varrho: G / F \rightarrow H / F^{\varphi}$ inducing $\mu$ and satisfying $b_{1}^{\varrho}=v_{1}$. Now we choose the pairs $(x, \bar{x})$ in (16) and (20) such that $\bar{x}=x^{e}$. By 3.4 there exists a canonical basis $\bar{B}_{\mu}$ of $\mu$ containing $\left(b_{1}, v_{1}\right)$ and all these pairs $\left(x, x^{\varrho}\right)$. If $\psi^{*}$ is the $L$-embedding of $G$ in $H$ constructed via 3.6 using $\mu$ and these new $\varphi(M)$, then by (b) of 3.6 there exists a canonical basis $\mathbb{C}$ of $\psi^{*}$ which contains $\mathbb{B}$. In particular, $\psi^{*}$ is canonical and this proves the lemma.

PROOF OF THEOREM C. Suppose first that $G=A \times B \times C_{3} \times \cdots \times C_{r}$ has an $L$-embedding in $H$. Then by $2.3, H=A^{\varphi} \times B^{\varphi} \times C_{3}^{\varphi} \times \cdots \times C_{r}^{\varphi}$ where $A^{\varphi}$ and $B^{\varphi}$ are $k$-smooth of $k$-type $B^{\varphi}$ and $\left|C_{i}^{\varphi}\right|=\left|C_{i}\right|=p$ for all $i$. If $k=1$, we are done; and if $k \geq 2$, then $2.6-2.8$ show that (ii) or (iii) of Theorem $C$ holds accordingly as $V$ is or is not elementary abelian.

Conversely, assume that $H$ has the properties given in Theorem C. It suffices to show that $A \times B$ has a canonical $L$-embedding in $U \times V$; by [1, Theorem 3.1 and 3.3], this can be extended to $G=A \times B \times C_{3} \times \cdots \times C_{r}$. By 2.6-2.8, the conditions (i) (iii) imply that $V$ is smooth and $U$ is $k$-smooth of type $V$. Let $1=U_{0}<\cdots<U_{n}=U$ and $1=V_{0}<\cdots<V_{k}=V$ be $k$-smooth chains with $U_{i} \simeq V_{i}$ for $i=0, \ldots, k$; let $1=A_{0}<\cdots<A_{n}=A$ and $1=B_{0}<\cdots<B_{k}=B$. Then there exists a canonical $L$-embedding $\varphi$ of $A \times B$ in $U \times V$ satisfying $A_{i}^{\varphi}=U_{i}$ and $B_{j}^{\varphi}=V_{j}$ for all $i, j$; indeed, by induction, we have such an $L$-embedding of $A_{n-1} \times B$ in $U_{n-1} \times V$ which, by Lemma 3.9, can be extended to $A \times B$. This proves Theorem $C$.

\section{References}

[1] D. W. Barnes, 'Lattice embeddings of prime power groups', J. Austral. Math. Soc. 2 (1962), 17-34.

[2] C. Herrmann and P. Huhn, 'Zum Begriff der Charakteristik modularer Verbände', Math. Z. 144 (1975), 185-194.

[3] B. Huppert, Endliche Gruppen I (Springer, Berlin, 1967).

[4] R. Schmidt, Subgroup lattices of groups (De Gruyter, Berlin, 1994).

Mathematisches Seminar

Universität Kiel

D-24098 Kiel

Germany 